\title{
Article
}

\section{Impact of Inter-Gateway Distance on LoRaWAN Performance}

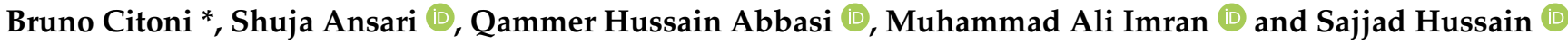 \\ James Watt School of Engineering, University of Glasgow, Glasgow G12 8QQ, UK; \\ shuja.ansari@glasgow.ac.uk (S.A.); qammer.abbasi@glasgow.ac.uk (Q.H.A.); \\ muhammad.imran@glasgow.ac.uk (M.A.I.); sajjad.hussain@glasgow.ac.uk (S.H.) \\ * Correspondence: b.citoni.1@research.gla.ac.uk
}

Citation: Citoni, B.; Ansari, S.;

Abbasi, Q.H.; Imran, M.A.; Hussain, S. Impact of Inter-Gateway Distance on LoRaWAN Performance. Electronics 2021, 10, 2197. https:/ / doi.org/10.3390/electronics10182197

Academic Editors: Jorge Navarro Ortiz, Cristina Cervelló-Pastor, Iván Vidal and Jasone Astorga

Received: 29 July 2021

Accepted: 6 September 2021

Published: 8 September 2021

Publisher's Note: MDPI stays neutral with regard to jurisdictional claims in published maps and institutional affiliations.

Copyright: (c) 2021 by the authors. Licensee MDPI, Basel, Switzerland. This article is an open access article distributed under the terms and conditions of the Creative Commons Attribution (CC BY) license (https:// creativecommons.org/licenses/by/ $4.0 /)$.

\begin{abstract}
The large-scale behaviour of LoRaWAN networks has been studied through mathematical analysis and discrete-time simulations to understand their limitations. However, current literature is not always coherent in its assumptions and network setups. This paper proposes a comprehensive analysis of the known causes of packet loss in an uplink-only LoRaWAN network: duty cycle limitations, packet collision, insufficient coverage, and saturation of a receiver's demodulation paths. Their impact on the overall Quality of Service (QoS) for a two-gateway network is also studied. The analysis is carried out with the discrete-event network simulator NS-3 and is set up to best fit the real behaviour of devices. This approach shows that increasing gateway density is only effective as the gateways are placed at a distance. Moreover, the trade-off between different outage conditions due to the uneven distribution of spreading factors is not always beneficial, diminishing returns as networks grow denser and wider. In particular, networks operating similarly to the one analysed in this paper should specifically avoid SF11 and 12, which decrease the average overall PDR by about $7 \%$ at $10 \%$ nodes increment across all configurations. The results of this work intend to homogenise behavioural assumptions and setups of future research investigating the capability of LoRaWAN networks and provide insight on the weight of each outage condition in a varying two-gateway network.
\end{abstract}

Keywords: LoRaWAN; low-power wide area network (LPWAN); Internet of Things; discrete-time simulation; stochastic geometry; uplink outage; quality of service; multi-gateway network

\section{Introduction}

The Internet of Things (IoT) is a term that defines electronic systems with sensors and actuators which are connected wirelessly. Such a network requires a way of transferring data between devices which has low power consumption, a high maximum range, and can potentially deal with a very large amount of wirelessly interconnected devices simultaneously [1]. One protocol that has gathered much attention in the past few years is LoRaWAN (Long Range WAN): a low power wide area network protocol (LPWAN) which satisfies the fundamental IoT requirements. The LoRaWAN framework is made up of two layers, the MAC layer and the PHY layer. The MAC layer, the proprietary LoRa (Long Range) technology, uses chirp spread spectrum (CSS) modulation to achieve long range communication. LoRa usually uses the licence-free, region-dependent ISM (Industrial, Scientific, and Medical) frequency bands: $863-870 \mathrm{MHz}$ for Europe and $902-928 \mathrm{MHz}$ for the US [2]. This makes it cheaper but also restricts the maximum achievable data rate because of regulations on available air time per device on those frequencies. This makes LoRa unsuitable for high data rate applications.

LoRaWAN networks are typically organised in a star-of-stars topology where end devices (nodes) do not have a direct connection to any single gateway but broadcast to all gateways in range, as shown in Figure 1. Each gateway relays the data it receives from all nodes in range to the network server it is associated with, where identical packets are de-duplicated. No handshake is required between nodes and gateways. This is in direct contrast to Wireless Sensor Networks, which are mostly organised in a mesh topology $[1,3]$. 


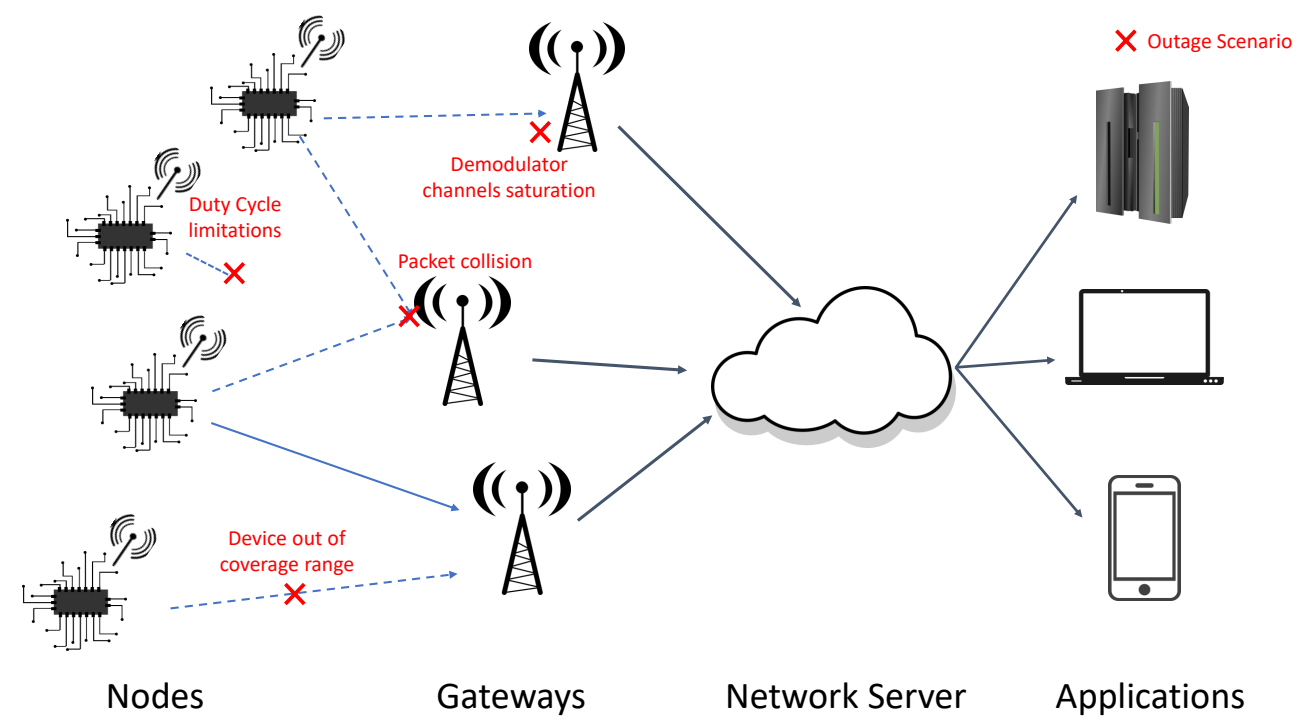

Figure 1. LoRaWAN star-of-stars network topology, including visual representation of the various outage conditions for an uplink-only network.

Communication is bidirectional, so devices can send data to the network via uplink and receive messages via downlink. Direct communication between two nodes is not available with standard LoRaWAN, requiring data to always pass through a gateway for both uplinks and downlinks. The gateways are restricted by the same duty cycle regulations on outgoing traffic as the nodes, so downlink messages are much rarer than uplink ones. Moreover, including downlink capabilities adds increased complexity in the network server design, increases the energy consumption per device, and lowers the overall QoS of a network by creating additional interferers. These limits preclude the use of LoRaWAN for time-critical, low-latency applications and have been extensively studied, especially those concerning its scalability [4]. To overcome some of these limits, a number of configurable parameters can be used to vary the maximum achievable communication range, power consumption, and data rate. The most important is the Spreading Factor (SF), commonly ranging from 7 to 12 . This relates to the number of chirps that are used to modulate the signal. Larger spreading factors increase the communication range, at the cost of increased energy consumption and longer air-time for each packet. Given the practical infeasibility of setting up large-scale LoRaWAN deployments, to help analyse their performance, both stochastic geometry models [5-9] and discrete-time simulations $[10,11]$ have been implemented across the literature. However, these studies lack a cohesive behavioural model and a comprehensive list of the possible outage conditions that a packet in an uplink-only network has to survive to be correctly received and decoded. Generally, due to the mathematical complexity of modelling a thoroughly accurate network, most research dealing with stochastic geometry limits the scope of the analysis to simpler, less realistic configurations, usually by reducing the number of variables. Moreover, none of these studies tries to compare their results with discrete-time packet level simulations, such as the ones performed with NS-3 or LoRaSim.

We have developed a model for the discrete-time simulator NS-3 to assess the relative impact of each of the known outage conditions on a two-gateway network. This was achieved by evaluating the Packet Delivery Ratio (PDR) of various network configurations upon varying parameters, such as the number of nodes deployed, the area they are deployed over, and the distance between the two gateways used.

The contributions of this paper are twofold:

- First, we develop a comprehensive list of the known outage conditions in a practical, uplink-only, LoRaWAN network. 
- Then, using the NS-3 discrete-time simulator, the weight of each outage condition on the overall QoS of a two-gateway LoRaWAN network is also assessed, as a factor of the inter-gateway distance.

The rest of this paper is structured as follows: first, we bring forward the known outage conditions and discuss the relevant literature in Sections 2 and 3. We then describe the simulation settings in Section 4 before discussing the results in Section 5. Finally, the outcomes of this work are summarised in Section 6.

\section{LoRaWAN Outage Conditions}

Before highlighting the limits of the current literature, it is appropriate to first define the various outage conditions that can affect a LoRaWAN packet in an uplink-only network.

All the following conditions need to be true during the lifetime of a packet to achieve correct transmission and decoding, as shown in Figure 1. A single failure will cause the data to be lost, either by dropping a packet, not decoding it properly, or not sending it in the first place. As such, they should all be accounted for in any LoRaWAN model or simulation.

\subsection{Duty Cycle Limitations}

In the EU, the enforced duty cycle is $1 \%$ for the commonly used frequency sub-bands of $863.00-868.00 \mathrm{MHz}$ and $868.00-868.60 \mathrm{MHz}$ [4]. Each sub-band must remain "silent" for a period of time that is proportional to the time-on-air of the last transmitted packet and the maximum available duty cycle enforced, according to the following [4]:

$$
T_{\text {silence }}=T_{\text {air }}\left(\frac{1}{d c}-1\right)
$$

where:

$T_{\text {silence }}=$ time-of-silence required after transmission;

$T_{\text {air }}=$ time-on-air;

$d c=$ duty cycle.

The time-on-air of a LoRaWAN packet can be calculated via the following [12]:

$$
T_{\text {air }}=T_{\text {preamble }}+T_{\text {payload }}
$$

with $T_{\text {preamble }}$ and $T_{\text {payload }}$ being the time duration for the preamble and the payload, respectively.

$$
T_{\text {preamble }}=\left(N_{\text {preamble }}+4.25\right) \times T_{\text {sym }},
$$

with $N_{\text {preamble }}$ being the number of programmed preamble symbols, 8 for the UK [13], and $T_{\text {sym }}$ being a symbol's duration.

$$
T_{\text {payload }}=p d_{\text {sym }} \times T_{\text {sym }},
$$

with $p d_{\text {sym }}$ being the number of symbols for the header and the payload.

$$
\begin{gathered}
T_{s y m}=\frac{2^{S F}}{B W} \\
p d_{\text {sym }}=8+\max \left(\left\lceil\frac{2 P L_{b}-S F+11-5 H}{S F-2 D E}\right\rceil \times(C R+4), 0\right),
\end{gathered}
$$

with:

$\max =$ the maximum operator, selecting the highest value between the two inputs;

$P L_{b}=$ number of payload bytes, including 13 additional bytes if header is present;

$S F=$ Spreading Factor;

$H=0$ if header is present, or 1 if not;

$D E=0$ or 1 , depending on whether low data rate optimisation is present; 
$C R=$ Coding Rate from 1 to 4 (being $4 / 4+C R$ ).

As the transmission interval between packets $(T)$ is set up by the designer, transmitting above the duty cycle limit is usually disregarded as a possible outage condition. However, if an adaptive duty cycle algorithm is enabled, a device might end up trying to transmit over its duty cycle limit after switching to a higher SF. For instance, a device that was set to transmit a packet of 10 bytes every $90 \mathrm{~s}$ on a single sub-band would be working as expected on SF7 with an air time of $61.7 \mathrm{~ms}$ and $T_{\text {silence }}$ of $6.1 \mathrm{~s}$ (Equations (1) and (2)) but would be over its duty cycle limit if set to SF12 without adjusting the transmission interval, with an air time of now $1482.8 \mathrm{~ms}$ and $T_{\text {silence }}$ of $146.8 \mathrm{~s}$. This could result in what is essentially "invisible" packet loss. Most LoRa transceivers are set up to abide to this limit, so the transmission would be stopped on the node by the chip itself [14]. The data would not be queued up for retransmission unless the device's algorithm was specifically designed to do so. This behaviour can be manually overwritten, but doing so could violate the law if transmitting above the duty cycle limit.

\subsection{Device out of Coverage Range}

The coverage probability of a node is determined by the device location, the sensitivity of the SF used for transmission and the propagation loss of the network. The sensitivity of a receiver can be calculated with the following [12]:

$$
S=-174+10 \log _{10} B W+N F+S N R,
$$

where the bandwidth $(B W)$ is typically $125 \mathrm{kHz}$, the noise figure $(N F)$ between $3 \mathrm{~dB}$ and $6 \mathrm{~dB}$ [6], and the SNR depends on the SF of the transmission, according to Table 1.

The propagation model used in the simulations performed in this paper is the logdistance propagation model [15]:

$$
P L=P L_{0}+10 \gamma \log _{10} \frac{d}{d_{0}}+X_{g}
$$

with:

$P L_{0}(\mathrm{~dB})$ being the path loss at the reference distance $d_{0}$, calculated using the Friis free-space path loss model;

$d$ is the distance between the transmitter and the receiver;

$d_{0}$ is the reference distance;

$\gamma$ is the path loss exponent;

$X_{g}$ is a variable that can be used to model slow and fast fading.

Temporarily ignoring the effect of interference and fading, the packet will suffer outage if:

$$
P_{t x}-P L \leq S_{s f}
$$

with:

$P_{t x}$ is the power of the transmission, $14 \mathrm{dBm}$, the maximum allowed by legal limits in the $\mathrm{UK}[16]$;

$P L$ is the path loss as defined above;

$S_{s f}$ is the sensitivity of the receiver for the specific SF of the transmission, based for this work on the values in Table 1. 
Table 1. Parameters for a network with a single gateway placed at the origin, using the log-distance path loss model, ignoring shadowing and fading

\begin{tabular}{|c|c|c|c|c|}
\hline SF & $S N R_{\min } @ R x(d B)^{a}$ & $S_{G W}(d B)^{b}$ & $\mathbf{R}_{\max }(\mathrm{m})^{\mathrm{c}}$ & $\operatorname{AoD}\left(\mathrm{km}^{2}\right)^{\mathrm{d}}$ \\
\hline 7 & -7.5 & -124.5 & 3011 & 28 \\
\hline 8 & -10 & -127 & 3509 & 10.2 \\
\hline 9 & -12.5 & -129.5 & 4089 & 13.84 \\
\hline 10 & -15 & -132 & 4766 & 18.83 \\
\hline 11 & -17.5 & -134.5 & 5554 & 25.54 \\
\hline 12 & -20 & -137 & 6473 & 34.72 \\
\hline
\end{tabular}

a Minimum SNR at the receiver, from Reference [17]. ${ }^{\mathrm{b}}$ Gateway Sensitivity, using Equation (7). ${ }^{\mathrm{c}}$ Maximum distance from a gateway that a node at each SF can be placed at, using Equation (10). ${ }^{\mathrm{d}}$ Area of deployment for nodes operating on each SF, based on $R_{\max }$.

\subsection{Demodulator Channels Saturation}

A practical fail case in commercially available gateways is the saturation of the receiver's demodulation channels. Packet reception involves a gateway "locking on" a packet preamble and decoding it, provided the RX power satisfies the sensitivity conditions of the receiver. If the packet is received, this process will occupy one of the demodulating paths of the receiver for a time that is proportional to the air time of the packet, plus a delay that is based on the distance between the node sending the packet and the gateway receiving it. If all demodulating paths are occupied by packets being decoded, the next packet arriving will not be received. In commercially available gateways, there are usually 8 parallel demodulators [18]. The probability of channel saturation depends on packets air time, nodes location and density, the nodes transmission interval, and the capacity of the network gateways.

\subsection{Packet Collision}

Finally, under the assumption of perfect orthogonality between different spreading factors, each packet will experience interference from packets sent at the same time, on the same frequency, and using the same SF. However, it has been proven that the quasiorthogonality of the different SFs can have a big impact on the overall network quality and scalability, with each packet also experiencing interference from signals sent at the same time, on the same frequency, but on a different SF [19].

Because of the capture effect, a packet can still be received over this interference if it achieves a minimum power difference from the strongest interferer. These thresholds have been modelled according to the work of Reference [20]. They show how a packet will be received over another on the same $\mathrm{SF}$ if the first is at least $6 \mathrm{~dB}$ stronger than the second.

More recent research conducted by Reference [19] shows that different thresholds are obtained with an experimental setup. Here, only $1 \mathrm{~dB}$ separation is needed for the strongest packet to be correctly received over the total interference on the same SF.

The overall chance of packet collision is affected by the nodes' location and density, as well as their SF, packet transmission interval, and packet air time.

\section{Related Works}

The impact of increased gateway density and their positioning in LPWANs has been extensively studied in literature. In Reference [21], the authors perform an analysis of optimal placement of 16 and 25 gateways with different strategies, and show that, while 25 gateways generally outperform 16, using a placing algorithm based on Fuzzy C-Means can boost the performance of 16 gateways to match that of 25 gateways. In Reference [22], both a practical and a theoretical method are provided to find the optimum location of gateways in a transmit-only network.

An overview of different LoRaWAN simulators is given in Reference [23], along with details of their native capabilities, though crucially not considering the ability to model the demodulators' saturation outage condition. In Reference [24], the authors develop 
the LoRaSim simulator and use it to confirm that LoRa networks scale well if dynamic transmission parameters selection or additional gateways are introduced. Similarly, in Reference [10], the authors develop NS-3 modules for simulating LoRaWAN and propose a scalability analysis, including different SF assignment methods, multiple gateway scenarios, and retransmission algorithms. However, they ignore the channel saturation outage condition. Another approach is proposed in Reference [25], where the focus is shifted onto the power consumption of the nodes, with the creation of NS-3 modules able to model it alongside accurate collision behaviour. However, both the quasi-orthogonality of different SFs and the limit of having a finite number of demodulator paths available at the receiver are not taken into account. Finally, in Reference [11], the authors develop their own NS-3 modules to demonstrate how a gateway can ideally support up to $10^{4}$ nodes and how increasing the number of gateways so that every gateway covers $6 \mathrm{~km}^{2}$ can improve the coverage probability of a node to above $90 \%$. This model is highlighted in Reference [23] as one of the best possible to perform accurate LoRaWAN analysis.

A number of papers have also analysed the problem of scalability by creating mathematical models for LoRa networks using stochastic geometry. In Reference [26], the authors perform a thorough study of collision and frame loss in a network including the possibility of re-transmissions, though they do not extend the analysis to other possible outage conditions. In Reference [5,6], Georgiou et al. model a single gateway network, with uniform and non-uniform node distributions, respectively. These analyses assume perfect orthogonality between different SFs, ignoring the effect of inter-SF interference. They also neglect the effects of the demodulator channel saturation on the overall outage probability. The same scenario, with the same limitations, is expanded to include a second gateway in Reference [7]. In Reference [9], the authors provide a similar setup, but this time incorporating the effect of quasi-orthogonality of the spreading factors, and using the collision matrix calculated in Reference [19]. This work again ignores the practical limitation of a finite number of parallel demodulation paths on the receiver. To the best of our knowledge, this outage condition is only taken into consideration for simulation purposes by Reference [8]. This paper analyses a single gateway network, limiting the concurrent demodulation paths available to 8 , while assuming perfect SF orthogonality. This outage condition, along with collision behaviour, is also validated via a series of different experimental deployments carried out by the authors in Reference [27]. This work, however, fails to address the duty cycle as a possible cause of outage in case an adaptive duty cycle algorithm is active at the network server. Moreover, this study is an experimental validation of the specifications provided by SEMTECH for LoRaWAN, without a formal analysis either in terms of stochastic geometry or discrete time simulation. Given the number of variables involved in modelling a realistic network, most research models simplify the underlying LoRaWAN assumptions to keep the complexity of the resulting analysis low. With our simulation model, we tried to recreate an uplink-only network's behaviour as faithfully as possible.

\section{System Model and Simulation Setup}

The simulation is performed in NS-3, using a modified version of the modules developed by Magrin et al. [11].

At the core of the simulation is the propagation model. This is the log-distance model, approximating the losses in an urban environment. The parameters for $\gamma, P L_{0}$, and $d_{0}$ are the ones used by Magrin et al. [11] and are in Table 2.

To calculate the maximum distance a node can be placed from any gateway, no fading is considered. Only the relative distance between a node and a gateway is used to calculate the overall power loss, using the log-distance model. By inserting the parameters into Equation (8) and solving for $d$, the maximum distance a signal of a given SF can be successfully decoded at can be found with the following:

$$
d \leq 10^{\frac{P_{t x}-P L_{0}-X_{g}-S}{10 \gamma}} d_{0} .
$$


The results for the different gateway sensitivities for each SF, using the parameters found in Table 2, are in Table 1.

Table 2. Simulation parameters.

\begin{tabular}{cc}
\hline Payload Length & $\mathbf{1 0}$ bytes \\
\hline$C R$ & $4 / 5$ \\
$B W$ & $125 \mathrm{kHz}$ \\
Channel & $868.3 \mathrm{MHz}$ \\
Tx Power & $14 \mathrm{dBm}$ \\
Sim. Time & $3600 \mathrm{~s}$ \\
Tx Time & $180 \mathrm{~s}$ \\
$P L_{0}$ & $7.7 \mathrm{~dB}$ \\
$d_{0}$ & $1 \mathrm{~m}$ \\
$\gamma$ & 3.76 \\
Noise Figure (NF) & $6 \mathrm{~dB}$ \\
Rodes $(N)$ & $500,1000,2000,5000$ \\
Radius $(R)$ & $3011,3509,4089,4766,5554,6473 \mathrm{~m}$ \\
Dimce between GWs $(D)$ & $125,0.25,0.375,0.5,0.625,0.75,0.875,1$ \\
\hline
\end{tabular}

The path loss and fast fading along with the total interference due to inter- and intra-SF collisions are all used to determine whether a packet is successfully delivered and decoded. The payload of each packet is set to 10 bytes, which, together with the 13 mandatory LoRaWAN header bytes, makes it so the time-on-air for a SF12 transmission will be $1482.8 \mathrm{~ms}$, as per Equation (2) [12]. The time between transmissions, $T$, is set for all nodes to $180 \mathrm{~s}$, so to allow for the $1 \%$ duty cycle restrictions to be satisfied for any SF, according to Equation (1). The overall simulation time is set so that each node will send a total of 20 packets throughout the run. The SF allocation is the standard distance-based allocation method. Each node is assigned the lowest spreading factor that still achieves a good link with at least one gateway. No Adaptive Data Rate (ADR) algorithm is applied, meaning that the SF does not change for any node and remains what it was set up to be as the simulation started. In addition, no downlink transmissions or re-transmission algorithms are taken into consideration. The rejection matrix proposed by Reference [20] is used to check for packet collisions. Finally, modelling the limitations due to the finite demodulation paths on a receiver, each gateway can "lock on" to, at most, 8 packets at a time.

The simulation is performed by varying the total number of nodes $N=\{500,1000$, $2000,5000\}$, the radius the nodes are uniformly distributed on $R=\{3011,3509,4089,4766$, $5554,6473\}$ metres and the distance between the two gateways as a factor of the network radius $D=\{0,0.125,0.25,0.375,0.5,0.625,0.75,0.875,1\}$, with 0 being the centre of the network and 1 being the whole radius length on either side of the origin, i.e., for $D=0.5$, the two gateways will be placed at $0.5 \times R$ on either side of the deployment origin, giving an inter-gateway distance of $R$; see Figure 2.

The gateway distances $(D)$ were chosen to give a good spread of possible inter-gateway distances, as was the total amount of nodes $(N)$, which reflect typical values found in literature $[9,28]$.

The radius $(R)$ values were chosen to be as close as possible to the calculated "boundaries" between SF. For instance, every node transmitting in a network with a single gateway, operating under the parameters we have configured, will be able to operate at SF7 if the network has radius $R=3011 \mathrm{~m}$. Conversely, in the same network but with radius $R=6473 \mathrm{~m}$, all SFs will have to be used, with SF8 being selected for devices in the first annulus bound by $3011 \mathrm{~m}<R<3509 \mathrm{~m}$, and so on. 


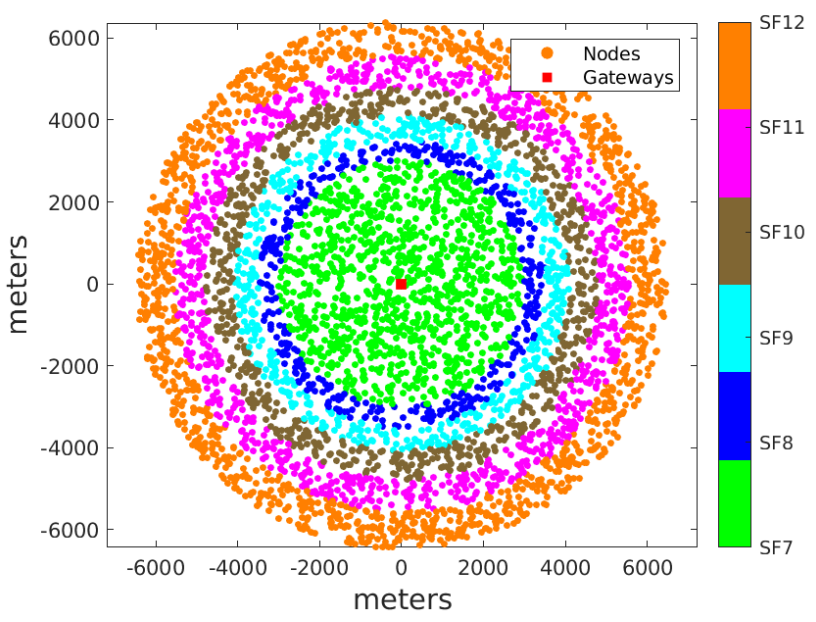

(a)

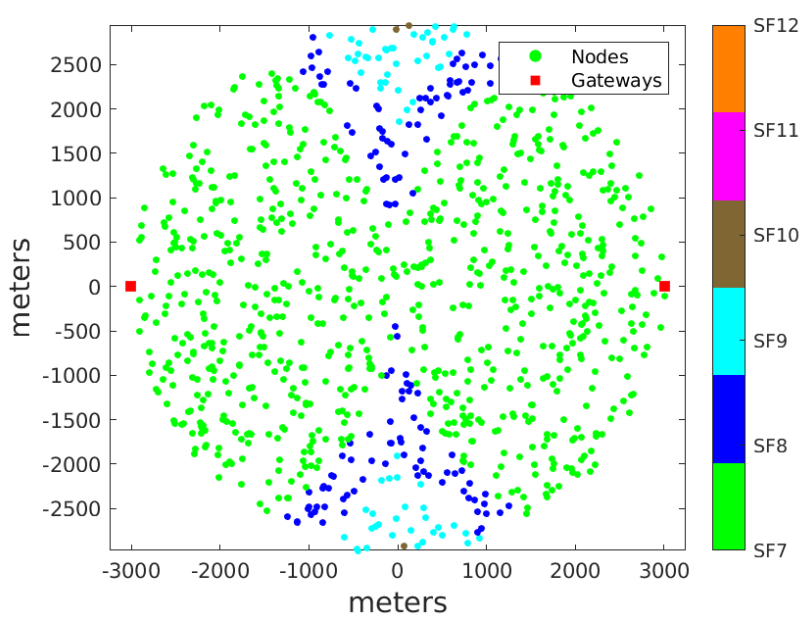

(b)

Figure 2. Two examples of network topologies, showcasing the different SFs distributions with varying network parameters $N, D$, and $R$. (a) 2 gateways, $N=5000, D=0, R=6473 \mathrm{~m}$. (b) Two gateways, $N=2000, D=1, R=3011 \mathrm{~m}$.

This is no longer true for a two-gateway system. Having calculated that the maximum distance a single gateway can cover is a circle of radius $6473 \mathrm{~m}$ around itself, it will be impossible in a network with nodes distributed over a radius of $6473 \mathrm{~m}$ for the two gateways to be placed anywhere else than the origin and cover the whole deployment area. This is illustrated in Figure 3.

The maximum distance between gateways is calculated with

$$
z=\sqrt{\frac{R_{\text {max }}^{2}-R_{d}^{2}}{R_{d}^{2}}},
$$

with:

$R_{\text {max }}=$ maximum distance a node can be from a gateway on a given SF, from Table 1; $R_{d}=$ radius of the network deployment.

Results for $z$ for the different network radii are given in Table 3 . This represents the maximum distance between two gateways that can guarantee all nodes will be within range of at least one gateway. We then only perform simulations with $D \leq z$ to ensure this. As an example, for simulations with $R=6473 \mathrm{~m}$, only $D=0$ is taken into consideration; for those with $R=4766 \mathrm{~m}$, every value, except for $D=1$, can be used. 


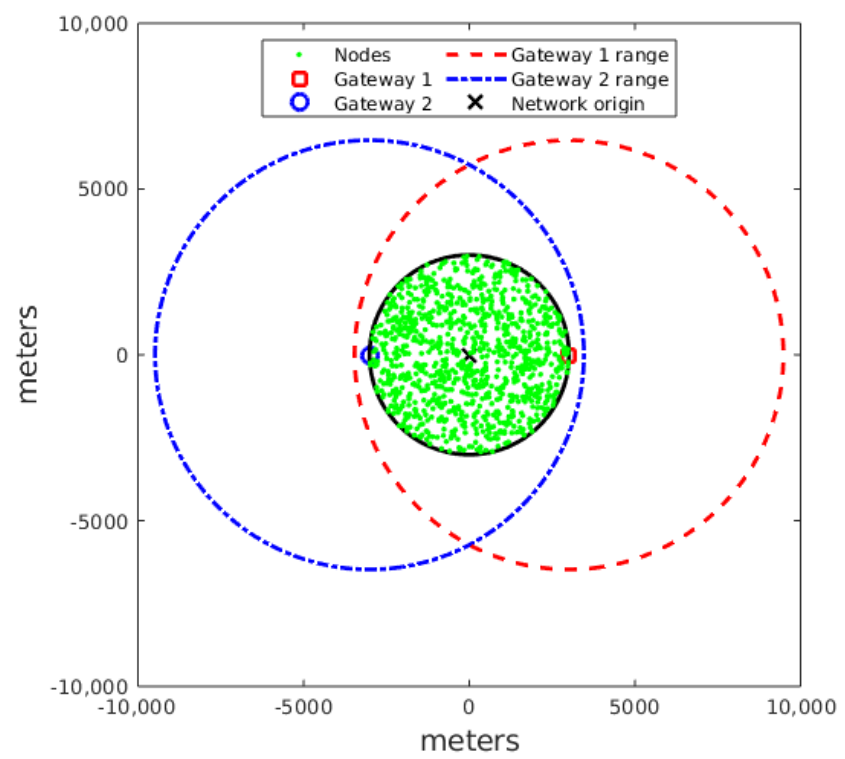

(a) $D=1, R=3011 \mathrm{~m}$

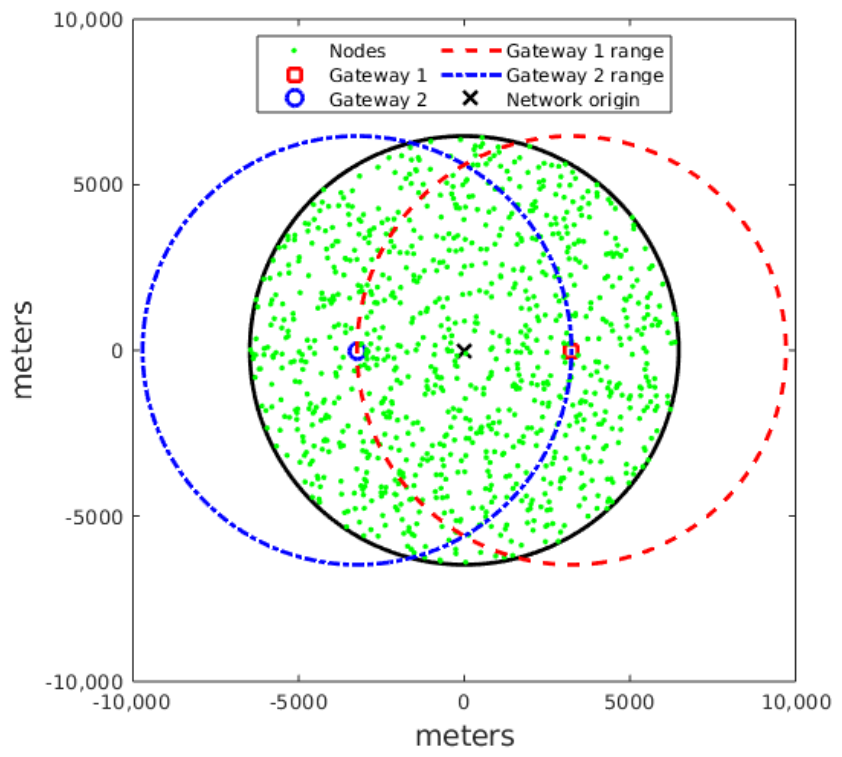

(b) $D=0.5, R=6473 \mathrm{~m}$

Figure 3. Examples of different gateway distance $(D)$ and network radius ( $R$, black circle) scenarios. (a) All nodes deployed are covered by both gateways. (b) A portion of the deployed nodes are not covered by either.

The whole simulation is first performed as described, specifically ignoring any fast fading effects, and then performed again, this time including fast fading. The fast fading is modelled using the Nakagami-m model with $\mathrm{m}=1$, to equate the Rayleigh distribution [29], and simply concatenated with the path loss already described in the NS-3 simulator.

The simulation results, obtained with NS-3, were further analysed and plotted through MATLAB. 
Table 3. Maximum distance of a symmetrical pair of gateways from the centre of the deployment so that all devices will be covered by the combined coverage of the two gateways, $z$.

\begin{tabular}{cccc}
\hline $\mathbf{S F}$ & $\mathbf{R}_{\mathbf{m a x}}(\mathbf{m})$ & $\mathbf{z}$ & Range of Valid $\boldsymbol{D}$ for Full Coverage \\
\hline 7 & 3011 & 1.9 & $0-1$ \\
8 & 3509 & 1.55 & $0-1$ \\
9 & 4089 & 1.22 & $0-1$ \\
10 & 4766 & 0.91 & $0-0.875$ \\
11 & 5554 & 0.59 & $0-0.5$ \\
12 & 6473 & 0 & 0 \\
\hline
\end{tabular}

\section{Simulation Results}

Key metric to quantify the QoS of a LoRaWAN network is its Packet Delivery Ratio (PDR), which provides an estimate of the quantity of data that is successfully transmitted and decoded, with a higher PDR meaning a network where fewer packets are dropped. This is formally defined as

$$
P D R=100 \times \frac{\text { packetsReceived }}{\text { packetsSent }}
$$

Results in Figure 4 show how increasing the number of nodes or increasing the network radius has the general effect of lowering the overall PDR. This result is true across all possible setting combinations, and it is a widely documented limitation of LoRaWAN scalability [4]. Similarly, increasing the network radius also decreases the overall PDR across all configurations, with nodes having to switch to higher spreading factors to stay within reach of a gateway, thus increasing the chance of collision. More interestingly, the results show how increasing the distance between two gateways generally increases the PDR. The benefits of increasing gateway density are well understood in literature for both LPWAN and LoRaWAN, with a direct correlation between number of gateways and PDR $[10,11,24]$.

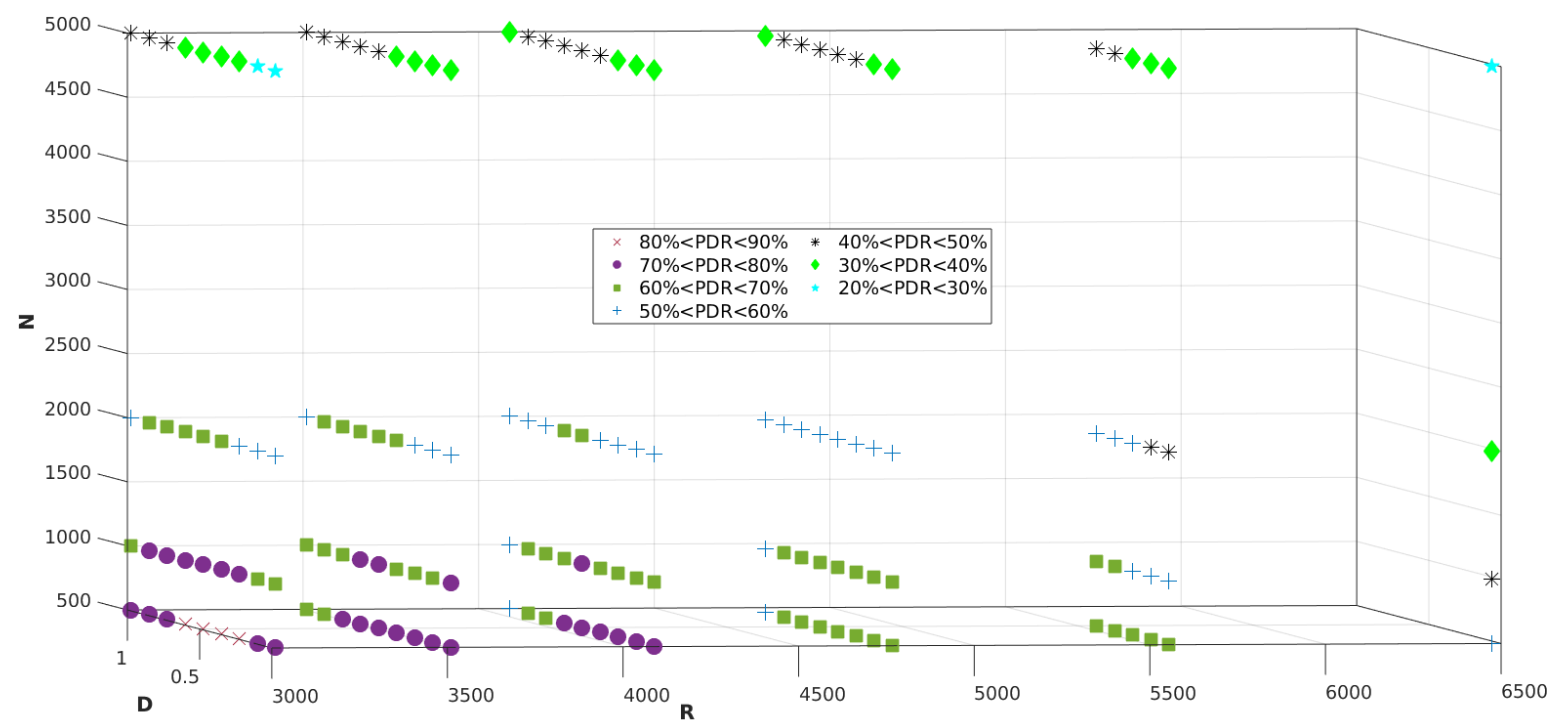

Figure 4. Three-dimensional scatter plots for the PDR across all simulations with fast fading enabled performed in this study, highlighting how low $D$ simulations have lower PDR compared with higher $D$ with otherwise identical configuration.

However, in a LoRaWAN network, this benefit is also heavily dependent on the gateway positioning, due to specific features, such as the star-of-stars topology and the capture effect. In an unrealistic scenario where two identical gateways with identical antennas are placed in the same location $(D=0)$, the second gateway would contribute nothing towards the PDR of the network. In LoRaWAN, packets delivered to multiple gateways are 
de-duplicated by the network server and have no inherent use except redundancy in case of gateway failure. This is clear from Figure 4 where lower distances between gateways $(D)$ tend to yield lower PDR relative to the same configuration. A similar drop in PDR can also be noticed for the largest $D$ values in most configurations, making values of $D$ around 0.5 generally the best for the same settings. This decrease in the overall PDR when excessively distancing gateways can be explained by looking at the effects this has on all possible outage conditions, as is shown in Table 4.

Table 4. Breakdown of the weight of different outage conditions on a selected range of network configurations, all with fast fading enabled.

\begin{tabular}{cccccc}
\hline $\boldsymbol{N}$ & $\boldsymbol{R}$ & $\boldsymbol{D}$ & Under Sensitivity Loss\% & Collision Loss\% & Ch. Saturation Loss\% \\
\hline 500 & 3011 & 0 & 14.17 & 7.95 & 0 \\
500 & 3011 & 1 & 27.91 & 1.94 & 0 \\
5000 & 3011 & 0 & 24.1605 & 49.8485 & 0 \\
5000 & 3011 & 1 & 41.585 & 16.522 & 0 \\
500 & 4089 & 0 & 21.395 & 4.255 & 0 \\
500 & 4089 & 1 & 38.41 & 1.64 & 0 \\
5000 & 4089 & 0 & 31.8035 & 31.291 & 0.0445 \\
5000 & 4089 & 1 & 47.639 & 13.258 & 0.044 \\
500 & 6473 & 0 & 31.825 & 10.605 & 0 \\
5000 & 6473 & 0 & 30.697 & 23.1245 & 24.2625 \\
\hline
\end{tabular}

The number of packets under sensitivity increases as the two gateways are positioned further apart. This is because more nodes will be able to transmit with a lower SF as a result of the gateways diverging from the network's origin. As a result, packets from some of the nodes that decreased their SF will only be received by the closer of the two gateways, making it more likely that interference and fast fading will cause outage.

This has the opposite effect on the collision probability. As the distance between the two gateways increases, the probability of collision decreases. This is because of the introduction of higher SFs in the network. In networks where all nodes can transmit at SF7, for instance, the chance of collision will be high, but, by moving the gateways apart, higher SFs will have to be introduced, hence decreasing this probability. This is visible in Figure $2 \mathrm{~b}$, where moving the two gateways to $D=1$ introduces SF8, 9, and 10 to a network that, with both gateways placed at the origin, would consist of SF7 only.

Channel saturation only becomes an issue for networks with a great number of nodes $(\geq 2000)$ and with a radius, such as higher SFs $(\geq 11)$, are needed. In the case of Figure $5 b$, with a $6473 \mathrm{~m}$ radius and more than 2000 nodes, it is shown that this kind of loss also rises much more sharply than the others. In this case, it appears that the inter-gateway distance can be increased provided the resulting distance between nodes and either gateway allows them to transmit with an SF not greater than 10.

Overall, results in Table 4 and Figure 5a show how the trade-off between different losses makes it beneficial to introduce higher SFs in a network operating under the assumptions described in Section 4, having low node density and low overall network radius. However, this benefit quickly disappears and turns into a hindrance as the network deviates from this configuration.

For a network radius of $R=3011 \mathrm{~m}$, it can be seen that, with $N=5000$, increasing $D$ from 0 to 1 increases the "under sensitivity" loss by roughly $17 \%$ but decreases the collision probability by $35 \%$, giving a better overall PDR for $D=1$. For a network radius of $R=4089 \mathrm{~m}$ and the same number of devices, $\mathrm{N}=5000$, increasing $D$ from 0 to 1 has no additional effect on the difference between the two "under sensitivity" loss percentages, which stays around $16 \%$. However, now the collision probability does not decrease as much by increasing $D$ from 0 to 1 , with a decrease of just $18 \%$. 


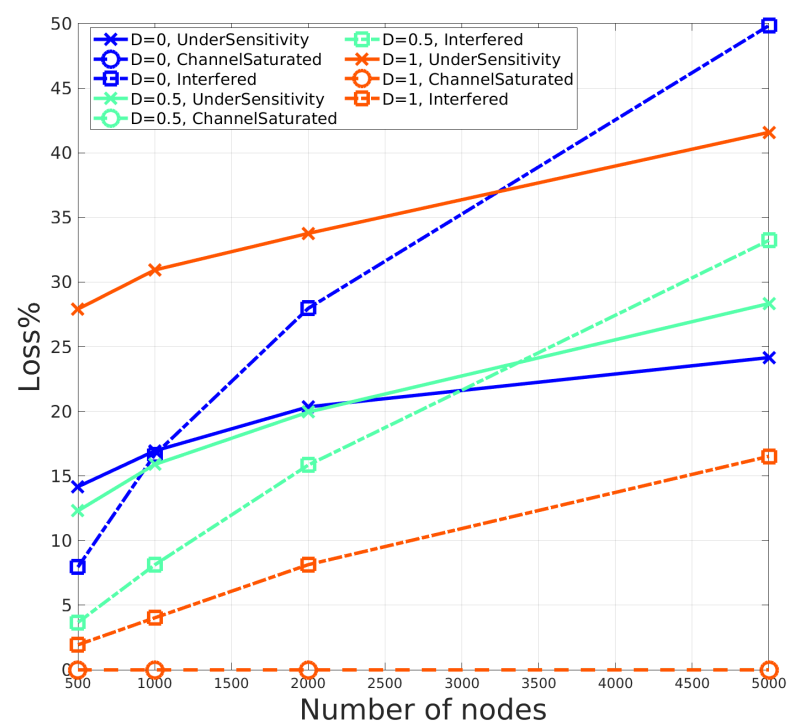

(a)

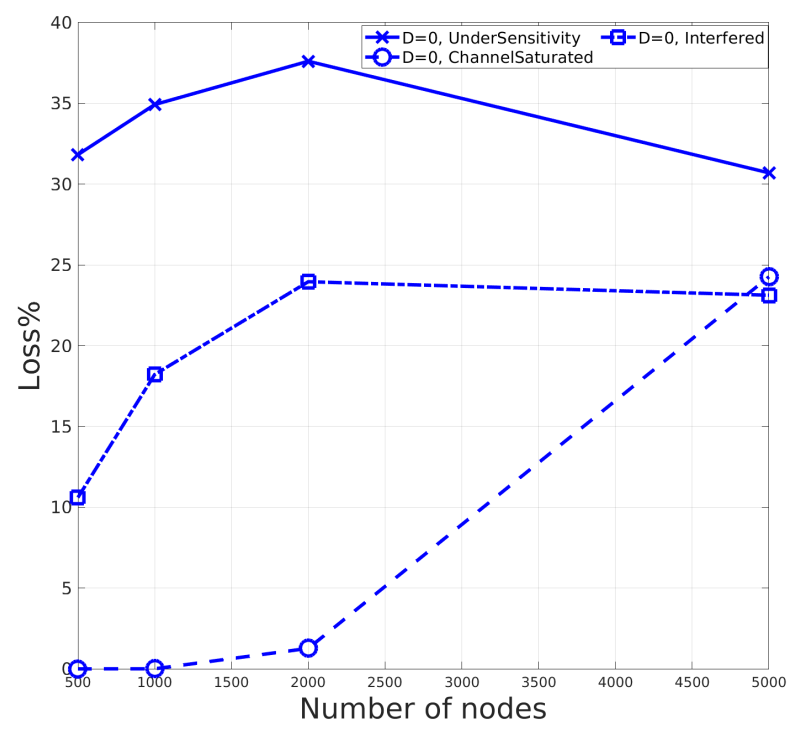

(b)

Figure 5. Total loss percentages for the different outage conditions across multiple $N$, with fast fading enabled. (a) $R=3011 \mathrm{~m}$. (b) $R=6473 \mathrm{~m}$.

By raising the chances of collision (inter- and intra-SF) and channel saturation due to the elongated time-on-air, the negative effects SF11 and 12 have on the PDR of similar dense networks are greater than the benefits they introduce by reducing the chance of "under sensitivity" outage and same SF collision. This is contrary to the intuitive idea that establishing the strongest possible link between a node and a gateway is always favourable, as confirmed in Reference [30]. Figure 6 depicts the lines of best fit through all simulations performed for this study. Increasing the percentage of nodes operating at any given SF is especially bad on the average PDR for SF11 and 12, while the increasing slope of the SF7 line shows that, ideally, a network operating with the setup described in this work is best set up with as many nodes operating at SF7 as possible. 


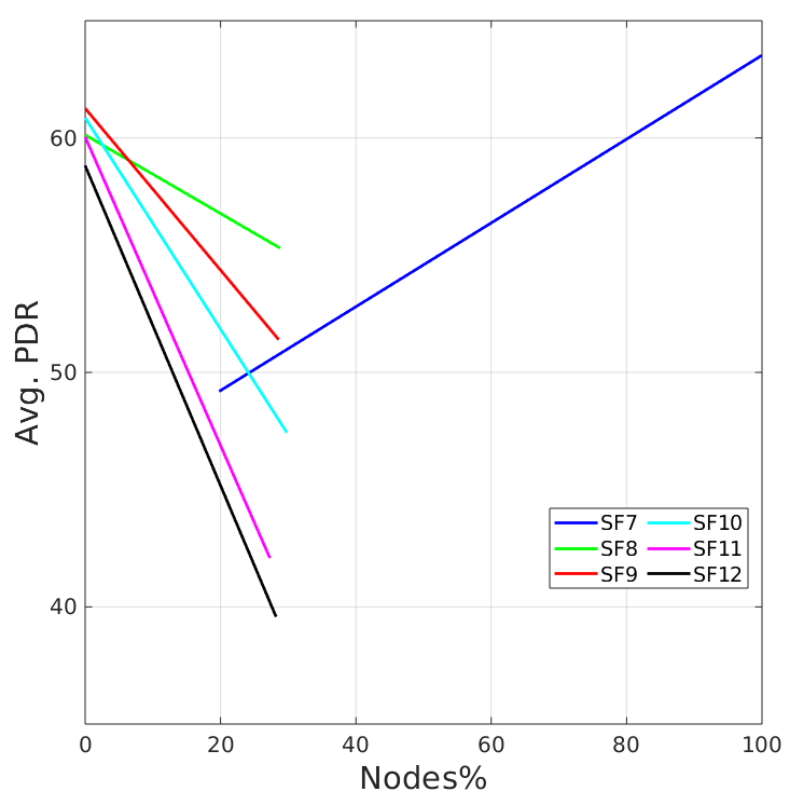

Figure 6. Average PDR of a whole network as a function of the percentage of nodes operating at each SF in the same network, with fast fading enabled.

This is not always possible, but SF11-12 should never be selected for such networks, either by keeping the deployment size small enough that they are not needed, by increasing the number of gateways or by developing appropriate and more involved SFs allocating algorithms [28,31].

Following these results, it appears that, in a network operating in a similar fashion, with equivalent device density and distribution, similar and fixed $T$, and the same SF allocation method, gateways are best placed at a distance that is roughly equal to the network radius, $R$, and equidistant from each other. Furthermore, the SF allocation method based on spreading all the possible SFs to nodes seems to be detrimental for larger and denser networks operating under an equivalent setup as the one described in this study. In such networks, the goal should be to minimise as much as possible the amount of nodes transmitting with larger SFs. As we have shown, the QoS of a similar network decreases rapidly as more devices operating at SF11 and 12 are added, with an approximate loss of $7 \%$ PDR for each $10 \%$ increment on the amount of nodes operating with those SFs.

\section{Conclusions}

Taking as a reference a network with two gateways, a correlation between their relative distance and the network QoS was established via NS-3. This is not only regarding the network's overall PDR, but to the weight of each outage condition in an uplink-only scenario. In particular, to maximise the PDR, two gateways should not be placed either too close or too far, and ideally, for a similar deployment, at a distance of about a whole network radius. The known outage conditions were also catalogued in a comprehensive way for easy reference to homogenise behavioural assumptions for future research investigating the capability of LoRaWAN networks, as well as informing real-life network designers. In future work, the scope of the problem will be expanded upon by including additional factors, such as downlink traffic, variable packet transmission delay, the introduction of multiple channels, and frequency hopping, as well as packet re-transmission algorithms. The impact of all these theoretical improvements on scalability should also be coupled with an energy consumption analysis. This would make sure that the best trade-off between performance and power can be achieved when translating research into real life deployments. 
Author Contributions: Conceptualization, B.C.; formal analysis, B.C., S.H. and Q.H.A.; software, B.C. and S.A.; validation, B.C., S.A., S.H. and Q.H.A.; visualization, B.C.; writing-original draft preparation, B.C., S.H. and Q.H.A.; writing, review, and editing, B.C., S.H., Q.H.A. and M.A.I. All authors have read and agreed to the published version of the manuscript.

Funding: This research was funded by UK EPSRC grant number EP/R512266/1.

Data Availability Statement: Data available on request.

Acknowledgments: The authors would like to thank the UK EPSRC supporting Bruno Citoni PhD studentship and to acknowledge the support of the wider Communication Sensing and Imaging (CSI) research group, School of Engineering, University of Glasgow.

Conflicts of Interest: The authors declare no conflict of interest.

\section{References}

1. Augustin, A.; Yi, J.; Clausen, T.; Townsley, W.M. A study of Lora: Long range \& low power networks for the internet of things. Sensors 2016, 16, 1466. [CrossRef]

2. Centenaro, M.; Vangelista, L.; Zanella, A.; Zorzi, M. Long-range communications in unlicensed bands: The rising stars in the IoT and smart city scenarios. IEEE Wirel. Commun. 2016, 23, 60-67. [CrossRef]

3. Sanchez-Iborra, R.; Cano, M.D. State of the art in LP-WAN solutions for industrial IoT services. Sensors 2016, 16, 708. [CrossRef] [PubMed]

4. Adelantado, F.; Vilajosana, X.; Tuset-Peiro, P.; Martinez, B.; Melia-Segui, J.; Watteyne, T. Understanding the Limits of LoRaWAN. IEEE Commun. Mag. 2017, 55, 34-40. [CrossRef]

5. Georgiou, O.; Raza, U. Low Power Wide Area Network Analysis: Can LoRa Scale? IEEE Wirel. Commun. Lett. 2017, 6, 162-165. [CrossRef]

6. Georgiou, O.; Psomas, C.; Skouroumounis, C.; Krikidis, I. Optimal Non-Uniform Deployments of LoRa Networks. IEEE Wirel. Commun. Lett. 2020, 9, 1919-1923. [CrossRef]

7. Georgiou, O.; Psomas, C.; Krikidis, I. Coverage Scalability Analysis of Multi-Cell LoRa Networks. In Proceedings of the IEEE International Conference on Communications, Dublin, Ireland, 7-11 June 2020. [CrossRef]

8. Sørensen, R.B.; Razmi, N.; Nielsen, J.J.; Popovski, P. Analysis of LoRaWAN Uplink with Multiple Demodulating Paths and Capture Effect. In Proceedings of the IEEE International Conference on Communications, Shanghai, China, 20-24 May 2019. [CrossRef]

9. Mahmood, A.; Sisinni, E.; Guntupalli, L.; Rondon, R.; Hassan, S.A.; Gidlund, M. Scalability Analysis of a LoRa Network under Imperfect Orthogonality. IEEE Trans. Ind. Inform. 2019, 15, 1425-1436. [CrossRef]

10. Abeele, F.V.; Haxhibeqiri, J.; Moerman, I.; Hoebeke, J. Scalability analysis of large-scale LoRaWAN networks in ns-3. IEEE Internet Things J. 2017, 4, 2186-2198. [CrossRef]

11. Magrin, D.; Centenaro, M.; Vangelista, L. Performance evaluation of LoRa networks in a smart city scenario. In Proceedings of the IEEE International Conference on Communications, Paris, France, 21-25 May 2017. [CrossRef]

12. SEMTECH. Designer 's Guide; SEMTECH. 2013; pp. 1-9. Available online: https://www.rs-online.com/designspark/relassets/ds-assets/uploads/knowledge-items/application-notes-for-the-internet-of-things/LoRaDesignGuide.pdf (accessed on 6 September 2021)

13. LoRa Alliance. LoRaWAN ${ }^{\circledR}$ Regional Parameters 1.1; LoRa Alliance. 2021. Available online: https://lora-alliance.org/wpcontent/uploads/2020/11/lorawan-regional-parameters-v1.1ra.pdf (accessed on 6 September 2021).

14. Microchip. RN2483; Microchip. 2019; pp. 1-22. Available online: http://ww1.microchip.com/downloads/en/Appnotes/AN307 6-Adding-LoRa-RN2483-Click-to-AVR-IoT-WG-Board-00003076A.pdf (accessed on 6 September 2021).

15. Gaussian Waves. Log Distance Model. Available online: https://www.gaussianwaves.com/2013/09/log-distance-path-loss-orlog-normal-shadowing-model/ (accessed on 6 September 2021).

16. Ofcom. IR 2030-UK Interface Requirements 2030; Ofcom. 2021. Available online: https:/ /www.ofcom.org.uk/_data/assets / pdf_file/0028/84970/ir-2030.pdf (accessed on 6 September 2021).

17. Semtech Corporation. SX127x Wireless \& Sensing Products Datasheet; SX1276/77/78/79 2020. Available online: https://semtech. my.salesforce.com/sfc/p/\#E0000000JelG/a/2R0000001Rbr/6EfVZUorrpoKFfvaF_Fkpgp5kzjiNyiAbqcpqh9qSjE (accessed on 6 September 2021).

18. Semtech Corporation. SX1308 Wireless \& Sensing Products Datasheet; Semtech Corporation. 2017; pp. 1-40. Available online: https://semtech.my.salesforce.com/sfc/p/\#E0000000JelG/a/44000000MDnb/XsCTHcePJiAjE.V2EEZt3Wqu005Yf3 FBWbtF0A6Yv70 (accessed on 6 September 2021).

19. Croce, D.; Gucciardo, M.; Mangione, S.; Santaromita, G.; Tinnirello, I. Impact of LoRa Imperfect Orthogonality: Analysis of Link-Level Performance. IEEE Commun. Lett. 2018, 22, 796-799. [CrossRef]

20. Goursaud, C.; Gorce, J.M. Dedicated networks for IoT: PHY / MAC state of the art and challenges. EAI Endorsed Trans. Internet Things 2015, 1, 150597. [CrossRef] 
21. Matni, N.; Moraes, J.; Rosario, D.; Cerqueira, E.; Neto, A. Optimal Gateway Placement Based on Fuzzy C-Means for Low Power Wide Area Networks. In Proceedings of the 2019 IEEE Latin-American Conference on Communications, LATINCOM, Salvador, Brazil, 1-13 November 2019. [CrossRef]

22. Tian, H.; Weitnauer, M.A.; Nyengele, G. Optimized gateway placement for interference cancellation in transmit-only LPWA networks. Sensors 2018, 18, 3884. [CrossRef] [PubMed]

23. Marais, J.M.; Abu-Mahfouz, A.M.; Hancke, G.P. A Review of LoRaWAN Simulators: Design Requirements and Limitations. In Proceedings of the 2019 International Multidisciplinary Information Technology and Engineering Conference, Vanderbijlpark, South Africa, 21-22 November 2019; pp. 1-6. [CrossRef]

24. Bor, M.C.; Roedig, U.; Voigt, T.; Alonso, J.M. Do LoRa Low-Power Wide-Area Networks Scale? In Proceedings of the 19th ACM International Conference on Modeling, Analysis and Simulation of Wireless and Mobile Systems (MSWiM'16), Malta, 13-17 November 2016; ACM: New York, NY, USA, 2016; pp. 59-67. [CrossRef]

25. To, T.H.; Duda, A. Simulation of LoRa in NS-3: Improving LoRa Performance with CSMA. In Proceedings of the IEEE International Conference on Communications, Shanghai, China, 20-24 May 2018; [CrossRef]

26. Muthanna, M.S.A.; Wang, P.; Wei, M.; Abuarqoub, A.; Alzu'bi, A.; Gull, H. Cognitive control models of multiple access IoT networks using LoRa technology. Cogn. Syst. Res. 2021, 65, 62-73. [CrossRef]

27. Liando, J.C.; Gamage, A.; Tengourtius, A.W.; Li, M. Known and unknown facts of LoRa: Experiences from a large-scale measurement study. ACM Trans. Sens. Netw. 2019, 15. [CrossRef]

28. Cuomo, F.; Campo, M.; Caponi, A.; Bianchi, G.; Rossini, G.; Pisani, P. EXPLoRa: Extending the performance of LoRa by suitable spreading factor allocations. In Proceedings of the 2017 IEEE 13th International Conference on Wireless and Mobile Computing, Networking and Communications (WiMob), Rome, Italy, 9-11 October 2017; pp. 1-8. [CrossRef]

29. NS-3. Nakagami Model. Available online: https://www.nsnam.org/doxygen/classns3_1_1_nakagami_propagation_loss_model. html (accessed on 6 September 2021).

30. Sanchez-Iborra, R.; Sanchez-Gomez, J.; Ballesta-Viñas, J.; Cano, M.D.; Skarmeta, A.F. Performance evaluation of lora considering scenario conditions. Sensors 2018, 18, 772. [CrossRef] [PubMed]

31. Cuomo, F.; Gamez, J.C.C.; Maurizio, A.; Scipione, L.; Campo, M.; Caponi, A.; Bianchi, G.; Rossini, G.; Pisani, P. Towards traffic-oriented spreading factor allocations in LoRaWAN systems. In Proceedings of the 2018 17th Annual Mediterranean Ad Hoc Networking Workshop, Med-Hoc-Net 2018, Capri Island, Italy, 20-22 June 2018; pp. 1-8. [CrossRef] 